\title{
Semantic-Based Policy Engineering for Autonomic Systems
}

\author{
David Lewis ${ }^{1}$, Kevin Feeney ${ }^{1}$, Kevin Carey ${ }^{1}$, \\ Thanassis Tiropanis ${ }^{2}$, and Simon Courtenage \\ ${ }^{1}$ Trinity College Dublin \\ \{Dave.Lewis, Kevin.Feeney, Kevin.Carey\}@cs.tcd.ie \\ ${ }^{2}$ Athens Information Technology Centre \\ ttiraait.edu.gr \\ ${ }^{3}$ University of Westminster \\ courtes@westminster.ac.uk
}

\begin{abstract}
This paper presents some important directions in the use of ontologybased semantics in achieving the vision of Autonomic Communications. We examine the requirements of Autonomic Communication with a focus on the demanding needs of ubiquitous computing environments, with an emphasis on the requirements shared with Autonomic Computing. We observe that ontologies provide a strong mechanism for addressing the heterogeneity in user task requirements, managed resources, services and context. We then present two complimentary approaches that exploit ontology-based knowledge in support of autonomic communications: service-oriented models for policy engineering and dynamic semantic queries using content-based networks. The paper concludes with a discussion of the major research challenges such approaches raise.
\end{abstract}

\section{Introduction}

Autonomic Communications shares with the vision of Autonomic Computing the desire to develop systems that provide a level of self-configuration, self-optimization, self-healing and self-protection so freeing the human system administrator from having to understand the changing complexities of the distributed IT systems and networks [kephart03]. Autonomic Computing research has largely focused on the management of computing resources, i.e. information server farms, web servers, GRID applications, while Autonomic Communications studies the application of self management to the network management and control domains. The observation in this paper are inspired by research into autonomic ubiquitous computing, which highlights the challenges of extending human governance of systems to the wider user community and of the self-management of system that are highly heterogeneous and ad hoc. While focusing on the particular needs of autonomic communication, the analysis in this paper therefore focusses on the particular challenges of ubiquitous computing.

The need to provide dynamically adaptive management solutions, which can address the increasing complexity and scale of modern heterogeneous networks and 
adaptive distributed application services, is a fundamental challenge for autonomic system research. Policy Based Management (PBM) has emerged as an attractive approach for flexibly and dynamically controlling systems, services and network behaviour. In particular, over the last few years it has attracted increasing attention from researchers, industry and standards bodies (e.g. IETF, DMTF and TMForum). However, PBM suffer in two important respects. Firstly, as the managed system scales in complexity, it becomes increasingly complicated to determine the impact of policy changes on system behaviour. This problem arises due to the difficulty in linking policy models, which are usually expressed in specific policy languages, to suitable models of both elemental and emergent composite system behaviour. Secondly, current PBM systems are weak in resolving business- and user-level policies into enforceable system-level policies in a generic and automated way. Such interpretation and resolution usually requires expert mediation by a policy author with considerable domain knowledge. This approach becomes unsustainable as responsibility for the management of resources is increasingly delegated and decentralised, reflecting current organisational trends. The problem is further exacerbated as organisations become integrated with other (partner) organizations in e-commerce value chains, virtual organisations, Internet communities or collaborative projects between organisations. This results in significant increases in both the quantity and heterogeneity of the resources that must be managed by the human administrator.

Therefore a more systematic approach to the development and maintenance of policies is required, one which closely integrates the modelling of the managed system and its behaviour with capturing user goals and resolving them to systemexecutable policies. We call such a systematic approach policy engineering. In this paper we propose supporting the policy engineering process by using ontology-based semantic models of the managed system to enable automated reasoning about policy resolution and policy interactions. Such reasoning aims to ease the interaction between people involved in policy engineering for system goverance and the autonomic systems over which they have authority and responsibility.

Within the Semantic Web initiative it has been widely observed that ontological reasoning techniques will only provide true benefits once a sufficiently large body of semantically marked up content is available. Similarly in the context of autonomic management, ontology-driven policy engineering will only be of use in the context of autonomic systems where services and networks possess ontological representations. In the following sections we examine two scenarios where ontologies may play an important part in the functionality of services and networks, and can thus also motivate and support the use of ontology-based policy engineering approaches. The first scenario addresses the use of semantic service composition in ubiquitous computing environments (where a variety of service technologies exist, WSDL-based web services, GRID services, Jini, UPnP etc). The second addresses the use of ontology-based semantics in content-based networking for collecting information over heterogeneous systems. In both scenarios we will discuss the role that can therefore be played by ontology-based semantic models of the system in the use of policies for mediating between human stakeholders and the autonomic computing and communications systems involved. 


\section{Semantic Service Composition in Ubiquitous Computing Environments}

In this section we motivate our research into the problems faced in policy engineering by considering the management of ubiquitous computing systems. Such systems require user-driven, autonomous management solutions [weiser]. This domain provides a level of system heterogeneity and of distribution of policy authoring that focuses our investigation directly onto the problems emerging from applying PBM to complex enterprise and service provider networks. Such systems involve physical environments containing a number of devices and accompanying computing resources, which are all potentially networked via a variety of wireless network technologies.

A key characteristic of this domain is that elements can be dynamically combined in different ways to adaptively satisfy user requirements and operational context for a particular task. Users can roam between ubiquitous computing environments, encountering a wide range of computing resources and services, e.g. WLANs, climate controls, printers, as well as introducing resources of their own, e.g. laptops, PDAs, in-car systems and mobile phones. In managing such environments, therefore, very few assumptions can be made about the homogeneity of resources, of system behaviour or of users' rights, responsibilities and goals. Instead these have to be discovered and interpreted at runtime and dynamically resolved into resource management policies that can be enforced on the different elements currently in use.

The development of systematic techniques for policy engineering in such dynamic, heterogeneous application domains is hindered by the current range of different policy languages and variations in their expressive power. The latter is particularly restricted with respect to expressing the objects that make up the managed systems. Such object specifications typically provide incomplete descriptions of the system, for instance concealing the side effects of policy actions which may result in unexpected policydriven behaviour. Better tool support is required to handle heterogeneity when capturing and processing system behaviour for PBM.

We propose the use of ontologies to handle these issues in support of policy engineering. We adopt a service-oriented approach to describing the behaviour of systems, thus constraining the range of semantic expressions with which we have to deal. Ontology-based semantics are proposed by the Semantic Web initiative [berners-lee] to overcome some of the problems of heterogeneity and runtime discovery of capabilities in the WWW. Research has only recently begun into the use of ontology-based semantics to described services in the form of semantic web services [owls]. Such a Semantic Web based ontology approach will provide benefits to policy engineering in terms of increasingly widespread expertise, tool availability (e.g. editors, validators) and platform support (e.g. inference engines, repositories).

Our approach for defining semantic services for policy engineering extends that taken by the DAML community for OWL-S semantic web service language [owls]. This uses description logic based ontologies of the Web Ontology Language (OWL) [owl] to define the Inputs, Outputs, Preconditions and Effects of a service (often abbreviated to IOPE) as well as the resources used by that service. However we aim 
to extend the elementary resource model with a richer one suitable for integration with policy engineering. The premise of semantic web services is that the use of separately authored, but inter-changeable ontology models for describing IOPEs and resources allows inference engines, such as AI planners and matchmakers, to automate the discovery, composition, invocation and monitoring of services [mclraith]. The OWL-S specification, therefore, includes mechanisms for specifying the control and data flow constructs needed to define service compositions, reflecting many of the constructs already well established from the workflow and web service composition domains. This provides the highly adaptive model of the managed system needed for the ubiquitous computing domain, where automated service composition may dynamically generate adaptive applications tailored to a user's task requirements and operational context.

Policies, in their simplest form, are event-condition-action rules. They are regarded as being performed on behalf of subjects, i.e. the person or agent wishing to operate on a resource, and acting on a target, i.e. the resource upon which the subject seeks an operation. Policies have been employed in system access control, defining authorisation rules about whether a particular subject is permitted or denied access to a particular target resource [sloman]. Policies are also being increasingly applied to the management of IP network, e.g. for access control and QoS management [stone]. General purpose policy languages address both authorisation policies and obligation policies, the latter being rules about what and when a particular subject is required to do or not do to a particular target [damianou][uszok][kagal]. Policy languages assist administrators with the task of managing large policy rule sets through abstractions such as roles (used for grouping users) and domains (used for grouping subjects, targets and sub-domains). The engineering of policies is assisted through the reuse of policy specification elements, thereby also encouraging consistent understanding of similar policy rules.

Recent research has begun to exploit ontologies for more extensible expression of subjects and targets as well as exploiting existing inference engines to ease policy engineering problems such as policy authoring, de-confliction and distributed enforcement [tonti]. In our scenario, in order to address problems of detecting and resolving unwanted policy-driven behaviour in complex systems while at the same time automating support for resolving policies provided a wider range of users, the ontology-based approach to specifying policies must be combined with the managed service's semantic service models.

Problems in maintaining a set of accurate policies arise when the managed system's complexity grows beyond the ability of individual policy engineers. Typically this prompts the division of policy authoring between teams thereby increasing in the potential for policy conflicts. Some policy languages can detect and, in certain cases, resolve modal conflicts, e.g. authorisation policies simultaneously denying and permitting access by a particular user role to a particular target resource.

However, application specific conflicts are usually only detectable by manual inspection or on a case by case basis at runtime [lupu], and remain a major technical problem in the engineering of industrial scale policy sets. An administrator's knowledge of certain types of known conflicts can be captured as meta-policies which 
define rule execution precedents or prevent the authoring of potentially conflicting policies. However, where application specific policy conflicts have not been predicted, conflicts must be detected at runtime by exceptions at policy enforcement points. More problematically, concurrent policies executed at different policy execution points may result, possibly through ill-defined side effects, in mis-use or mis-allocation of resources in a way that is difficult to determine. Static detection of such problems is difficult and requires careful control and observation of different policy settings deployed across the system [dunlop].

In the ubiquitous computing scenario, the dynamic policy conflict detection problem is greatly exacerbated by the fact that the system itself changes greatly over time due to the large number of autonomous devices networking together in an ad hoc fashion to provide users with the application functionality they require. Such ad hoc collections may include devices and resources that users may temporarily bring to a particular ubiquitous computing environment, so a static pre-determination of available resources will be impossible to extract. Furthermore, user applications will not be statically bound to specific environments. Applications will be composed from service components in an ad hoc fashion in reaction to the user's current task requirements and other contextual information such as the user's location, social setting, schedule, authorisations, terminal display capabilities and wireless access links. Thus the authoring of application policies will suffer an even greater level of target system fluidity.

Finally, the fact that the application services and resources involved will be sourced from an un-limitable range of vendors will confound agreements on and conformance to standardised management models [osullivan].

Authoring management policies typically requires administrators to interpret natural language business rules and operational policies set down over time by organisational managers. Though it has been an oft stated objective of policy based management to automate the resolution of business goals into system-level policies, general purpose solutions to this problem are still the subject of active research [beigi][bandara]. This is, at least in part, due to the lack of a mechanism for providing the contextual knowledge needed to unambiguously interpret natural language business goals or user policies into a form that can be resolved into system-level resources.

Organisations own resources such as Wireless LAN, Internet access, email servers, file stores, and printers, the use of which administrators must ensure conforms with managerial goals. In addition, administrators (i.e. operational support staff) at wireless access network service providers will need to ensure that the key radio and network resources of ubiquitous computing environments are managed according to business goals implemented as admission control and QoS provision policies [murray]. Organisations are increasingly decentralising their business processes by flattening hierarchies and moving the responsibility for resource management decisions closer to the users of those resources. This results in policy authoring moving beyond just the sphere of specialised administrative staff and being given to individual team leaders and workers across the organisation. 
High-level policy languages allow subject and target specifications to be better aligned with concepts more familiar to the average user. However, few system and network management solutions natively support this level of abstraction, and so some bridging mechanisms are required [hull03a]. Where there is a wide heterogeneity in the elements of the managed system, the task to develop suitable bridging mechanisms becomes prohibitively large. This picture is exacerbated when we consider situations where teams with resource management responsibilities span organisational boundaries, involve individual contract workers or temporary visitors, or loose coalitions, such as many Internet-based communities.

Following the ubiquitous computing scenario to its full potential, application components and wireless resources will be adaptively assembled into disposable compositions on a per user, per task basis [chakraborty][masuoka]. The individual user, not wishing to be made aware of the details of each composition, may provide a set of preferences they wish to apply to their activities in general and to certain circumstances in particular, e.g. 'always select the cheapest available wireless link', 'only use secure applications for business tasks', 'always log activities to my home web site'. These preferences are effectively user defined policies that both control the resources they personally own, but which also can constrain the adaptive behaviour exhibited by the ubiquitous computing environments encountered to outcomes with which the user is comfortable.

These preferences need to be expressed in terms with which the user can relate, in particular in terms that relate to the tasks they wish to perform and the effectiveness or quality of service of the adaptive application generated by the ubiquitous computing environment to support this task [hull03b]. These user-defined policies need to be effectively resolved into system level policies and reconciled with the policies set by other users, teams and administrators responsible for the resources involved.

To establish a semantic service model that supports our policy engineering needs while remaining consistent with mainstream Semantic Web development we propose extending the OWL-S specification to improve the expression of the semantics for managed systems. We must therefore focus on specifying management semantics at two levels. One is at the level of the managed system as an application used by the user, which will typically involve composite services consisting of other composite and/or atomic services. The second level relates the resources used by services, which may participate in many concurrent service compositions.

OWL-S currently specifies a simplistic model of the resources that are used by a service and relatively few examples of its application exist. We propose extending the OWL-S resource ontology so that policies can specify, to varying degrees, how a service makes use of resources in various situations. We assert that the component developer is best placed to provide component-specific meta-policies restricting how service user or administrators can later apply policies controlling the deployed service's resource-related behaviour. In this way we can investigate how ontologybased semantics can be used to capture developer knowledge of a service component's use of resources and how this knowledge can be used in the engineering of system-wide policies. 
An initial structure for such an integration of service model, resource model and policy vocabulary centred on a deployable component is suggested in [lewis04a]. This represents a service component's managed behaviour as a rule-based automaton. This is in line with several models for ubiquitous computing components that express behaviour as rule sets [fitzpatrick][terada][owen]. Policy rules local to the service component can be set by the service administrator to govern how a particular deployment of the service component makes use of particular resources. Thus, administration of a service-oriented system is enforced by policies local to services components, rather than by policies that relate generally to the underlying resources. This makes the means of management consistent with the service-oriented principle that resources can only be accessed via well-defined services, in this case a policybased component management service. It also provides the means for a service component developer to design a more flexible component thus offering wider applicability through a common management mechanism.

Overall, the responsibility falls on the component developer to expose, via the extended semantic service specification, all the interactions between the service and the resources it uses, and to make appropriate control of these interactions possible via component-specific meta-policies. As behaviour rule sets can quickly become unmanageable we are currently examining the use of component behaviour ontologies based on finite state machines to expose just a selected subset of behaviour for policybased management purposes.

A further restriction of the OWL-S semantic service model is that, in common with many web service models, it is concerned only with the behaviour of application software functions, and does not model the behaviour of the networks that link them. From a management point of view, we are equally concerned with the behaviour of application software and of network resources, and therefore will need to include the latter in our semantic service model, thereby exposing their semantics for inclusion in end-to-end management, e.g. of performance, faults etc. Architectural Description Language (ADLs) [medvidovic] model systems as contracts, which are analogous to semantic services in their use of IOPEs, and connectors that enable contracts to communicate, while still possessing their own distinctive properties, e.g. representing the non-functional features of communication networks and middleware.

In ADLs, connectors are modelled as groupings of contracts. We can therefore model semantic connectors building on OWL-S in a way similar to semantic services, and in particular using the same policy based mechanisms for expressing how the implementation of a semantic connector interacts with the communication resources it uses. This will provide us with a single, simple mechanism for modelling a wide range of integrated service and network resources that may be dynamically configured for different applications. The connector abstraction also serves as the integration interface for more complex resource models that are not suited to expression using description logic ontologies.

In our ubiquitous computing application, composite service may consist of services and connectors supporting a number of resources on user terminals, wireless access networks and application services available in a particular environment. The service composition represents the semantics of the managed system to which user level 
policies need to be applied. By integrating the ability to enforce system level policies into the semantic descriptions of services and connectors, we are able to reason about differences in models within the policy resolution process. We can therefore exploit the semantics implicit in a service composition, linking the composite service and its IOPEs to the IOPEs of the constituent services, and thus, at the level of atomic service, to resource-level policies.

\section{Knowledge Based Networking}

The Semantic Web initiative has encouraged research into how ontology-based queries can be resolved in a distributed peer-to-peer manner between agents holding information with heterogeneous RDF-based semantics that are distributed over the web [cai][stuckenschmidt][tempich]. In [lewis04b] we outline a Semantic Query Based Network (SBQN) service that extends such distributed querying using Content Based Networks (CBN) to provide a Collaborative Information Service. CBNs use a publish-subscribe message delivery paradigm, but with message routing based on filters applied to message content. This provides more flexibility than routing based on a set of predefined message types [pietzuch][carzaniga].

The architecture for this service aims to securely and flexibly support the acquisition and dissemination of information between members of collaborative groups working across the Internet. It uses persistent ontology-based queries for defining the information being sought and shared, so that the range of supported application domains automatically reflects the ever-expanding range of domain ontologies that will be published for use in the Semantic Web. The SBQN service is also used to support the autonomic management and knowledge management needs of the network itself. The Collaborative Information Service used a SBQN to connect a number of web servers. Each server supports a different set of resource types, where resources are anything with a URI, the meta-data of which is described using OWL. Servers provide standard HTTP pull access to resources, and the SBQN supports push capabilities for resource meta-data. Permissions to advertise and subscribe to metadata are managed by the user community.

In the autonomic communication domain, we propose that the SBQN could be used for providing fine-grained dynamic collection of network status information. In the first instance such an application could use ontological models of existing network element MIBs [vergara], acting as a flexible management notification delivery service. However, the application of the SBQN becomes more interesting when extended to multiple administrative domains, including the user or customer domain and a dynamically formed chain of network and application service providers. Such situations may become increasingly dynamic as users may more easily select wireless access networks and wired backbone transport is commoditised and providers are switched dynamically. Impose on this an increase in customer applications that operate in the context of virtual organisation, then the need for flexible multiorganisational mechanisms to resolve access control policies on information mediated by the SBQN becomes apparent.

Role-based access control requires a priori agreement on roles and thus its use is limited in such fluid organisations structures. We have therefore established a more 
flexible abstraction for policy-based access control, where communities, rather than roles, become the central abstraction used to specify access control. In this case communities may represent resource management teams in organisations participating in a virtual organisation as well as the service provider administrators in any service provision chain they use. The power of this approach is that a community may mandate authority to both access resources and to author new access control policies for certain resources to a sub community. In this way the authoring of policies is distributed through a virtual organisation to the group considered best qualified to make those policies. An organisation thereby can organically grow and change, perhaps starting from a single group with all authority, and decomposing into subgroups with mandated authority based on specialisation of skills as the need arises naturally in the organisation's lifecycle.

Conflicts between policies authored by separate groups are automatically reported to the nearest mutual parent of the two groups. This group, having mandated policy authoring authority, is the best placed to decide how to resolve any resulting conflict. The resolution of such conflicts depends on a semantic model consisting of linked directed, acyclical graphs that represent the community structure, the authorisation dependencies between resources and the authorisation dependencies between actions that can be performed on those resources. Specific ontology-based models for these are currently being devised in alignment with the service and resource models outlined in the previous sections.

The semantics of the community-based policy abstraction and of how authority is mandated to sub-communities has been established [feeney]. A community policy management system has been implemented using Ponder, an existing policy based framework [damianou][sloman], and is found to operate satisfactorily. This implementation has been applied to the access control for a CVS code repository being used by an Internet community. Usage tests with that community are currently underway. This will provide us with initial user acceptance results with respect to the use of community-based access control.

\section{Discussion and Further Work}

These two scenarios presented above provide some motivation for why ontologybased semantics will be important in the modelling of adaptive networked systems, while also demonstrating how such semantics can potentially support more intelligent interaction between the people and autonomic systems. This extends the idea of a knowledge plane for the Internet [clark], to that of a semantic representation for services, for network links (as connectors) and for the possible constraints over adaptivity that can be imposed on the underlying resources. We thus emphasise the point that as autonomic management is essentially human governance resulting in the constraint of adaptive behaviour using policies, we must address the semantics of both adaptive networks and adaptive application software in relating such policies to the expected human experience.

This approach however, leaves many open questions relating to the limits of semantic based reasoning in the context of adaptive, networked systems. Currently standardised ontologies are based on description logic, which is soon to be 
complemented with semantic rules [horrocks]. Though the latter will obviously aid in the representation of policies using ontologies, it is far from clear that all forms of policy based management can be addressed with these ontological logics. For instance in [kephart04], policies are categorised into action policies, goal policies and utility logic. Though a combination of OWL and SWRL may go some way to being able to reason about the former (and then only with the support of ontologies for temporal logic), other logics suitable for feeding optimization algorithms may be needed. This in turn will require an extensible, modular structure for reasoners that is embedded in the network, similar to existing semantic application toolkits [oberle], but which is itself subject of autonomic management. For example, in the SBQN we envisage nodes dynamically subscribing with queries related to logic problems which they encounter, in order to locate suitable downloadable code to conduct the required reasoning. Equally, ontologies capturing mappings between concepts in separate domain ontologies that appear in user queries can also be sought and obtained by SBQN routing nodes using the SBQN service.

The SBQN architecture raises several issues that require further investigation in order to assess usability and scalability of this architecture for deployment on the Internet. We must perform a more detailed assessment of the performance possible with existing ontology-based matching algorithms, though in the long term we expect that optimised software and hardware support for OWL will emerge driven by its potential popularity, as has already happened for XML processing. One possible optimisation that will reduce the reasoning load on SQBN nodes will be to decompose ICS queries based on known routes prior to submitting them as subscription queries to the SQBN.

In general, further experimentation will be required to evaluate the scalability and performance of such knowledge based networking against variations in the numbers of information sources, sinks, advertisements, subscriptions and client joins/leaves. More challenging is the need to assess scalability against growth in the number and scope of ontology domains, ontology encoded logics and ontology mappings.

As pointed out in [barrett], within any realistic business scenario, policy authoring is a challenging collaborative activity. The community-based policy management approach we present goes some way to addressing the identification and resolution of conflicts between policies developed by different groups within fluid organisations, however the scheme does not yet exploit the full potential of ontology-based policy semantics for dealing with uncertainty about resources and identity as proposed in [kagal].

\section{References}

[bandara] Banadara, K., Lupu, E, Moffett, J., Russo, A., “A Goal-Based approach to Policy Refinement", in proc of 5th IEEE International Workshop on Policies and Distributed Systems and Networks, IEEE, 2004, pp 229-239

[barrett] Barrett, R., "People and Policies: Transforming the Human-Computer Partnership", in proc of 5th IEEE International Workshop on Policies and Distributed Systems and Networks, IEEE, 2004, pp 111-116 
[beigi] Beigi, M., Calo, S., Verma, D., "Policy Transformation Techniques in Policy-based Systems Management", in proc of 5th IEEE International Workshop on Policies and Distributed Systems and Networks, IEEE, 2004, pp 13-22

[berners-lee] Berners-Lee, T., Hendler, K., Lassila, O. (2001), 'The Semantic Web', Scientific American, pp 35-43, Issue 284 (3), 17th May 2001

[cai] Cai, M., Frank, M., "RDFPeers: A Scaleable Distributed RDF Repository based on a Stuructred Peer-to-Peer Network, in Proc. of World Wide Web Conference 2004, 17-22 May 2004, New York, NY, USA

[carzaniga] Carzaniga et al, "Design and Evaluation of a Wide-Area Event Notification Service”, In ACM Transactions on Computer Systems, Vol. 19(3), 2001, pp. 332-383.

[chakraborty] Chakraborty, D., Perich, F., Joshi, A., Finin, T., Yesha, Y., "A Reactive Service Composition Architecture for Pervasive Computing Environments", In 7th Personal Wireless Communications Conference (PWC 2002). Singapore. October. 2002.

[clark] Clark, D., Partridge. C., Ramming, J.C., Wroclawski, J.T. "A Knowledge Plane for the Internet", in Proc. of SIGCOMM'03, 25-29 August 2003, Karlsruhe, Germany

[damianou] Damianou, N., Dulay, N., Lupu, E., Sloman, M., (2001) “The Ponder Policy Specification Language", Proc. Policy 2001: Workshop on Policies for Distributed Systems and Networks, Bristol, UK, 29-31 Jan. 2001, Springer-Verlag LNCS 1995, pp. 17-28

[dunlop] Dunlop, N., Indulska, J., Raymond, K. (2003) "Methods for Conflict Resolution in Policy-based Management Systems", IEEE 7th International Enterprise Distributed Object Computing Conference (EDOC'03), Sept 16 - 19, 2003, Brisbane, Australia, pp 98-111

[feeney] Feeney, K., Lewis, D., Wade, V. "Policy-based Management for Internet Communities", in proc of 5th IEEE International Workshop on Policies and Distributed Systems and Networks, IEEE, 2004, pp 23-34

[fitzpatrick] Fitzpatrick, A., Biegel, G., Clarke, S., Cahill, "Towards a Sentient Object Model" In proceedings of the Engineering Context-aware Object-Oriented Systems and Environments workshop at OOPSLA 2002

[horrocks] Horrocks, I., Patel-Schneider, P., Boley, H., Tabet, S., Grosof, B., Dean, M. (2003), "SWRL: A Semantic Web Rule Language Combining OWL and RuleML", version 0.5, 19th November 2003, http://www.daml.org/2003/11/swrl/

[hull03a] Hull, R., Kumar, B., Lieuwen, D. (2003), “Towards federated Policy Management”, IEEE 4th International Workshop on Policies for Distributed Systems and Networks, June 04 - 06, 2003, Lake Como, Italy, pp 183-196

[hull03b] Hull, R., Kumar, B., Lieuwen, D., Patel-Schneider , P., Sahuguet , A., Varadarajan, S., Vyas, A. (2003), "Policy-based System for Personalized and Privacy-conscious User Data Sharing", Bell Labs Technical Memorandum, February, 2003.

[kagal] Kagal, L., Finin, T., Joshi, A., "A Policy Language for A Pervasive Computing Environment", IEEE 4th International Workshop on Policies for Distributed Systems and Networks, June 04, 2003

[kephart03] Kephart, J., Chess, D., “The Vision of Autonomic Computing”, IEEE Computer, Jan 2003, pp 41-50.

[kephart04] Kephart, J., Walsh, W., "An Artificial Intelligence Perspective on Autonomic Computing Policies", in proc of 5th IEEE International Workshop on Policies and Distributed Systems and Networks, IEEE, 2004, pp 3-12

[lewis04a] Lewis, D., Conlan, O., O’Sullivan, D., Wade, V. "Managing Adaptive Pervasive Computing using Knowledge-based Service Integration and Rule-based Behavior", in Proc. of 2004 IEEE/IFIP Network Operations and Management Systems, pp 901-902 
[lewis04b] Lewis, D., Feeney, K., Tiropanis, T., Courtenage, S., "An Active, Ontology-driven Network Service for Internet Collaboration", in Proc of Semantic Web for Web Communities workshop, 2004, Valencia, Spain.

[lupu] Lupu, E.C, Sloman, M. (1999), "Conflicts in Policy-Based Distributed Systems Management", IEEE Transactions on software engineering, vol. 25, no. 6, November 1999. pp 852-69.

[masuoka] Masuoka, R., Labrou, Y., Parsia, B., Sirin, E., "Ontology-Enables Pervasive Computing Applications”, IEEE Intelligent Systems, Sept/Oct 2003, pp 68-72

[mclraith] McIlraith, S.A., Son, T.C., Honglei Zeng, H. (2001), 'Semantic Web Services', IEEE Intelligent Systems, 16(2), March/April 2001

[medvidovic] Medvidovic, N. Taylor, R.N., (1997) "A framework for classifying and comparing architecture description languages", Proc. 6th. European Software Engineering Conference/Proc. 5th. Symposium on the Foundations of Software Engineering, Zurich, Switzerland, Sept. 1997, pp 60-76.

[murray] Murray, K., Mathur, R., Pesch, D., "Adaptive Policy-Based Access Management in Heterogeneous Wireless Networks", in Proc of IEEE WPMC, Vol. 1, pp 325-329, Oct 2003

[oberle] Oberle, D., Staab, S., Volz, R., "An Application Server for the Semantic Web", in Porc of WWW'04, May 17-22, 2004, New York, USA, pp. 220-221.

[osullivan] O'Sullivan, D., Lewis, D., "Semantically Driven Service Interoperability for Pervasive Computing", Proceedings of the 3rd ACM International Workshop on Data Engineering for Wireless and Mobile Access, San Diego, CA, USA, 19th September 2003, pp 17-24

[owen] Owen, T., Rathke, J., Wakeman, I., Watson, D, "Implementing Policies in Programs using Labelled Transition Systems Tim Owen Julian”, In Cosener's House Multi-Service Networks Conference, 2002, June 29, 2002

[owl] W3C (2003) Ontology Web Language, http://www.w3.org/2001/sw/, Visited Apr 2003

[owls] "OWL-S: Semantic Markup for Web Services", The DAML Service Coalition, http://www.daml.org/services/, October 2002

[pietzuch] Peter R. Pietzuch and Jean Bacon. Peer-to-Peer Overlay Broker Networks in an Event-Based Middleware. In H. Arno Jacobsen, editor, Proceedings of the 2nd International Workshop on Distributed Event-Based Systems (DEBS'03), ACM SIGMOD, San Diego, USA, June 2003. ACM.

[sloman] Sloman, M., Lupu, E., "Security and Management Policy Specification", IEEE Network, vol.16 No. 2, March/April 2002. pp 10-19.

[stone] Stone, G.N., Lundy, B., Xie, G.G. (2001), "Network Policy Languages: A survey and a new approach", Network, IEEE, Volume: 15 , Issue: 1, Jan.-Feb. 2001, pp10 - 21

[stuckenschmidt] Stuckenschmidt, H., Vdovjak, R., Houben, G.J., Broekstra, J., "Index Structures and Algorithms for Querying Distributed RDF Repositories", in Proc. of World Wide Web Conference 2004, 17-22 May 2004, New York, NY, USA

[tempich] Tempich, C., Staab, S., Wranik, A., "REMINDIN': Semantic Query Routing in Peerto-Peer Networks Based on Social Metaphors", in Proc. of World Wide Web Conference 2004, 17-22 May 2004, New York, NY, USA

[terada] Terada, Tsutoma, Tsukamoto, M., Hayakawa, K., Yoshihisa, T., Kishino, Y., Kashitani, A., Nishio, S., "Ubiquitous Chip: A Rule-Based I/O Control Device for Ubiquitous Computing”, in Proc Pervasive, 2004, LNCS 3001, pp. 238-253, 2004

[tonti] Tonti, G., Bradshaw, J.M., Jeffers, R., Montanari, R., Suri1, N., Uszok, A., "Semantic Web Languages for Policy Representation and Reasoning: A Comparison of KAoS, Rei, and Ponder" Proceedings of 2nd International Semantic Web Conference (ISWC2003), October 20-23, 2003, Sanibel Island, Florida, USA 
[weiser] Weiser, M. (1991), “The Computer of the 21st Century”, Scientific American, vol. 265, no.3, September 1991, pp 66-75.

[uszok] Uszok, A., Bradshaw, J., Jeffers, R., Suri, N., Hayes, P., Breedy, M., Bunch, L., Johnson, M., Kulkari, S., Lott, J. (2003), "KAoS Policy and Domain Services: Toward a Description-Logic Approach to Policy Representation, Deconflictions, and Enforcement", IEEE 4th International Workshop on Policies for Distributed Systems and Networks, June 04 - 06, 2003, Lake Como, Italy, pp 93-98

[vergara] de Vergara, J.E.L., Villagra, V.A., Berrocal, J., "Applying the Web Ontology Language to Management Information Definitions", IEEE Communications Magazine, special issue on XML Management, Vol. 42, Issue 7, July 2004, pp. 68-74. ISSN 0163-6804 